Artigo Original

Original Article

Ana Bassôa ${ }^{1}$

Adriana C. Costa ${ }^{2}$ (1)

Rudineia Toazza²

Augusto Buchweitz ${ }^{3}$ (1)

Descritores

Rastreio

Dislexia

Professores Escolares

Psicometria

Estudo de Validação

Keywords

Screening

Dyslexia

School Teachers

Psychometrics

Validation Study

Endereço para correspondência:

Augusto Buchweitz

Escola de Ciências da Saúde e da Vida,

Pontífícia Universidade Católica do

Rio Grande do Sul - PUCRS

Av. Ipiranga, 6690, Jardim Botânico,

Prédio 63, Porto Alegre (RS), Brasil,

CEP: 90610-000.

E-mail: augusto.buchweitz@pucrs.br

Recebido em: Abril 02, 2020

\section{Escala para rastreio de dislexia do desenvolvimento: evidências de validade e fidedignidade}

\author{
Scale for developmental dyslexia screening: \\ evidence of validity and reliability
}

\section{RESUMO}

Objetivo: Investigar validade e fidedignidade de uma escala de rastreio para dislexia do desenvolvimento (DD) no ensino fundamental preenchida por professores. Método: Avaliação empírica - 12 professores responderam a Escala de Leitura e Escrita (ELE) sobre 95 alunos de $3^{\circ}$ ano do ensino fundamental, em dois momentos; os escolares realizaram testes de leitura e escrita (variáveis externas) para investigar a correlação entre a escala e o desempenho dos mesmos. Realizaram-se (1) análise fatorial, (2) avaliação da consistência interna, (3) investigação da relação entre um item da escala e o construto medido por teoria da resposta ao item (TRI) (4) correlação da escala com variáveis externas (Validade Convergente-VC); e (5) investigação da estabilidade temporal da avaliação. Resultados: (1) a escala avalia um único fator; (2) o coeficiente alpha apontou que os itens são bons indicadores do construto; (3) a análise por TRI mostra que todos os itens foram monotônicos, indicando que um único construto determina a variabilidade (4) a correlação de Spearman foi moderada (11/17 itens), apontando a existência de VC; (5) o valor da correlação do coeficiente de estabilidade temporal indica que o resultado da ELE não varia de maneira significativa no tempo; (6) nove crianças obtiveram pontuações que sugerem encaminhamento para uma avaliação diagnóstica devido ao grau de dificuldade apresentado. Conclusão: $\mathrm{O}$ estudo mostra evidências empíricas de validade e fidedignidade da ELE para rastreio de risco de DD. A porcentagem de crianças com suspeita de DD (aproximadamente $9 \%$ ) corrobora a literatura internacional sobre prevalência de dislexia.

\begin{abstract}
Purpose: To investigate the empirical validity and reliability of a screener for risk of developmental dyslexia (DD) by elementary school teachers. Methods: The scale was tested with 12 teachers who answered questions about their students (95 students total, all in the third year of elementary school); the students, in turn, performed reading and writing tasks which were used to investigate the association between screening scores and performance. The following analyses were carried out: (1) factor analysis; (2) internal consistency; (3) relationship between each scale item and the construct of interest, as measured by item response theory (IRT); (4) correlation of each scale item with external variables (reading and writing tests); and (5) the temporal stability of teachers' evaluations. Results: The analyses showed: (1) one factor was extracted; (2) strong internal consistency - the items in the scale are good indicators for screening of this construct; (3) items were monotonic (IRT), i.e., item variability is associated with one construct; (4) moderate Spearman correlation (11/17 items); (5) temporal stability - the result of screening did not vary over time. Conclusion: This study shows evidence of validity and reliability of the proposed scale in its intended use of screening for developmental dyslexia. The percentage of children at risk for developmental dyslexia, according to the scale, was approximately $9 \%$, which is in agreement with the international literature on the prevalence of dyslexia.
\end{abstract}

Trabalho realizado na Pontifícia Universidade Católica do Rio Grande do Sul - PUCRS - Porto Alegre (RS), Brasil. ${ }^{1}$ Programa de Pós-graduação em Medicina e Ciências de Saúde, Neurociências, Pontifícia Universidade Católica do Rio Grande do Sul - PUCRS - Porto Alegre (RS), Brasil.

${ }^{2}$ Instituto do Cérebro do Rio Grande do Sul - InsCer - Porto Alegre (RS), Brasil.

${ }^{3}$ Escola de Ciências da Saúde e da Vida, Pontífícia Universidade Católica do Rio Grande do Sul - PUCRS Porto Alegre (RS), Brasil.

Fonte de financiamento: Coordenação de Aperfeiçoamento de Pessoal Nível Superior - Brasil (CAPES) Código de Financiamento 001 - $\mathrm{N}^{\mathrm{o}}$ do processo: 23038.002530/2-13-93.

Conflito de interesses: nada a declarar. 


\section{INTRODUÇÃO}

O objetivo deste estudo foi investigar evidências de validade e fidedignidade de uma escala de rastreio de sintomas da dislexia do desenvolvimento (DD), por meio de análise empírica. Esta escala, de autoaplicação, foi desenvolvida com o objetivo de ser de fácil acesso, uso e análise para professores e profissionais que trabalham com crianças em idade de alfabetização. Para tanto, projeta-se sua validade empírica e utilização no auxílio de identificação de sinais de risco para este transtorno de aprendizagem em face da ausência de instrumentos de rastreio de DD em português brasileiro.

ADD é um transtorno específico de aprendizagem de origem neurobiológica. Apresenta-se como dificuldades inesperadas para a idade cronológica, coeficiente intelectual e grau de escolaridade da criança, e que não podem ser explicadas por outro quadro diagnóstico ${ }^{(1)}$. Apesar do DSM-5 ${ }^{(1)}$ empregar o termo dislexia apenas na observação do Transtorno Específico da Aprendizagem com prejuízo da leitura, optou-se pelo termo dislexia do desenvolvimento pelo seu amplo e histórico uso.

Estima-se que a taxa mundial da dislexia se encontre entre $5 \%$ a $10 \%$ dos leitores. No Brasil, uma porcentagem de $7 \%$ totalizaria aproximadamente 3 milhões de disléxicos entre 49 milhões alunos da educação básica brasileira, no ano de 2014(2).

Apresenta-se o problema do diagnóstico da DD, no Brasil, onde a DD é um transtorno sub-diagnosticado ou diagnosticado tardiamente. Um estudo recente de avaliação de crianças brasileiras com dislexia identificou que $60 \%$ delas haviam sido reprovadas ao menos uma vez e que a idade média do diagnóstico ficava entre 10 e 11 anos o que sugere desinformação e ausência de rastreio para diagnóstico precoce ${ }^{(3)}$. Estas crianças já haviam cumprido entre 5 e 4 anos de escolarização sem que sequer um risco para DD fosse identificado a partir de seus sinais de risco, sendo que alguns destes sinais manifestam-se já na préescola ou no primeiro ano de ensino fundamental como aponta a literatura ${ }^{(4)}$.

A identificação precoce da DD mitiga a evasão e outros efeitos deletérios do baixo nível de leitura associado com o transtorno. Instrumentos de fácil aplicação para o rastreio de sinais de risco DD, como a presente Escala de Leitura e Escrita (ELE), auxiliam na investigação de sinais de risco. Ressaltase, nesse ponto, o papel do professor de ensino fundamental e a variedade de possíveis dificuldades de aprendizagem na escola pública atual. Estudos têm apontado a confiabilidade da capacidade do professor de julgar a habilidade de leitura de seus alunos ${ }^{(5)}$. A avaliação de desempenho de uma criança, comparada a seus colegas, pelo professor, e com um instrumento estruturado pode ser uma das estratégias para ajudar a resolver o problema de identificação precoce de crianças em risco para transtornos de aprendizagem.

A ELE pode identificar crianças que apresentam alguns sintomas e comportamentos característicos da DD. Enfatiza-se que a ELE não substitui a investigação clínica para diagnóstico da DD. A ELE foi construída com base na estrutura da escala para rastrear déficit de atenção e hiperatividade, SNAP-IV ${ }^{(6)}$ (Escala de Avaliação de Swanson, Nolan e Pelham) e com base em uma revisão teórica da literatura com enfoque, principalmente, nos manuais diagnósticos. Após esta primeira etapa, a ELE foi submetida a uma análise de juízes especialistas, de diferentes regiões do país. Esta análise levou à exclusão e inclusão de itens a partir da avaliação dos especialistas, bem com mudanças em seu texto visando deixar a escala inteligível e clara. O resultado deste processo foi exposto à análise de educadores de ensino fundamental para verificar se não haviam distorções na interpretação dos itens (análise semântica). Estas etapas e análises de construção da escala estão explicitadas em outro estudo ${ }^{(7)}$.

A escolha de validar no final do terceiro ano fundamenta-se no DSM-5(1). O manual estabelece, nos Critério C e D dos Transtornos Específicos de Aprendizagem, que as dificuldades devem aparecer durante os anos escolares e não podem decorrer das oportunidades educacionais. Com base nestes critérios e na Base Nacional Comum Curricular (BNCC), que estabelece que a alfabetização ocorra até o final do $3^{\circ}$ ano do ensino fundamental (quando é realizada a Avaliação Nacional de Alfabetização, por exemplo), compreendeu-se que, apenas a partir desta etapa, poder-se-ia estabelecer com maior precisão a hipótese diagnóstica de dislexia. $O$ terceiro ano, representa um marco das diretrizes para o processo de aprendizagem. Portanto, é no $3^{\circ}$ ano que as dificuldades de leitura provavelmente estariam reconhecidas pelos professores; é também, no Brasil, o primeiro ano em que se pode reprovar um aluno. Salienta-se que a prescrição da BNCC sobre "idade certa" é uma diretriz com embasamento em evidências sobre neurodesenvolvimento e melhor idade para aprendizagem da leitura ${ }^{(8,9)}$; entretanto, mesmo que arbitrária, esta prescrição estabelece um marco temporal no processo educacional brasileiro para o qual uma escala de rastreio pode auxiliar na identificação de risco para DD.

A ELE é única na sua natureza no Brasil. Encontrou-se apenas uma escala validada no país, que busca monitorar aspectos de desenvolvimento socioemocional como habilidades sociais, problemas de comportamento e competências acadêmicas de crianças de 6 a 13 anos (Inventário Habilidades Sociais, Problemas Comportamento e Competência Acadêmica para Crianças - SSRS $)^{(10)}$. Até o presente momento, não há escala equivalente que rastreie sinais indicativos de DD. Ressalta-se a originalidade e a necessidade de uma escala de rastreio desta natureza no país. A disponibilização deste instrumento de rastreio para sua utilização em diferentes regiões do país pode consolidar o uso da escala como um instrumento eficaz e simples de rastreio.

\section{TESTAGEM EMPÍRICA DE INSTRUMENTO: CONSISTÊNCIA INTERNA, ANÁLISE FATORIAL, TEORIA DE RESPOSTA AO ITEM, VALIDADE CONVERGENTE E COEFICIENTE DE ESTABILIDADE TEMPORAL}

A validade e fidedignidade de um teste possuem graus que são obtidos através de métodos pré-estabelecidos ${ }^{(11)}$ e que são utilizados na validação de instrumentos ${ }^{(12)}$. A seleção e construção dos itens do instrumento e demais indícios de validade encontram-se descritos no trabalho previamente mencionado ${ }^{(7)}$. No presente estudo, apresentam-se os resultados das avaliações estatísticas baseadas em dados empíricos, discutindo-se as implicações. 
Destacam-se as seguintes análises de validade da ELE e suas funções: a) consistência interna: testa se a variabilidade apresentada em cada item/tarefa possui forte relação com a variabilidade dos demais itens, e com a variação do escore final; b) análise fatorial: testa quantos comportamentos a ELE e seus itens avaliam, e o quanto cada item é uma boa representação do comportamento a que ele está relacionado; c) teoria da resposta ao item: testa a capacidade que cada item da escala tem de mensurar o grau de habilidade que cada sujeito apresenta; d) validade convergente: testa a correlação existente entre a variação do desempenho em provas que medem a habilidade desejada (variáveis externas) e a variação dos escores da ELE; e) coeficiente de estabilidade temporal: verifica a estabilidade da medida no tempo, estimando, assim, o erro de medida de quem preenche a ELE. Todos estes testes foram aplicados e a apresentação detalhada dos métodos de aplicação dos testes pode ser encontrada no trabalho prévio ${ }^{(7)}$. Apresentam-se aqui os resultados que embasam a validação empírica e uma discussão prática dos itens e sintomas que a ELE avalia.

\section{MÉTODO}

O presente estudo foi aprovado pelo Comitê de Ética em Pesquisa da Comitê de Ética em Pesquisa Pontifícia Universidade Católica do Rio Grande do Sul (CAAE número 51215715.8.0000.5336). Todos os professores que participaram e os responsáveis pelos alunos avaliados assinaram um termo de consentimento livre e esclarecido.

\section{Participantes}

A amostra infantil desta pesquisa foi oriunda de um projeto guarda-chuva (CAAE números 30895614.5.0000.5336 e 13629513.0.0000.5336). Para a avaliação das crianças com a ELE, o presente estudo contou com doze professoras (Tabela 1) de terceiro ano do ensino fundamental de seis escolas públicas responsáveis pelos escolares acompanhados pelo projeto. Estas escolas compõem uma amostra por conveniência do projeto guarda-chuva. As professoras avaliaram 122 crianças com a ELE. Vinte e sete avaliações foram excluídas por não terem preenchido os critérios de inclusão: a) criança com percentil $>25 \operatorname{RAVEN}(\mathrm{n}=13)$; b) ausência de avaliação intelectual $(n=6) ; c)$ avaliação de leitura e escrita incompleta $(n=7) ; d)$ protocolo incompleto pelo professor $(\mathrm{n}=1)$; portanto, a amostra final contou com 95 alunos (idade média 9,27 anos, DP =0,39; $52,6 \%$ feminino). A pesquisa foi realizada no ano escolar de 2015. Após um mês da primeira avaliação, todas professoras foram convidadas a participar da etapa de reteste. O tempo de resposta das professoras foi variado, de dois à quatro meses para a entrega dos protocolos. Ao final, seis professoras aceitaram participar do reteste. Nesta etapa, 30 crianças foram escolhidas aleatoriamente e reavaliadas (idade média 9,25 anos, $\mathrm{DP}=0,39$ ).

\section{Instrumentos e procedimentos}

A Escala de Leitura e Escrita - ELE é um instrumento autoaplicado (ver arquivo em anexo). Para a avaliação, utilizase uma escala likert de 4 pontos para mensurar a frequência com que os sintomas, elencados em 16 itens, se manifestam. A escala foi entregue aos professores com identificação dos alunos selecionados. A instrução foi de responder no prazo de quinze dias. A pontuação da escala foi a seguinte: cada item marcado com a frequência "nunca" recebia um ponto; "raramente", dois pontos; "às vezes", três pontos; e "frequentemente/sempre", quatro pontos. A pontuação mínima total, que indica nenhuma dificuldade, é 16 pontos; a máxima, que indica muita dificuldade, 64 pontos.

O desempenho de leitura e escrita foi avaliado em tarefas executadas em grupo e em sessões individuais. As tarefas utilizadas foram: a) Avaliação de leitura de palavras e pseudopalavras ${ }^{(13)}$; b) Avaliação da compreensão leitora de textos expositivos ${ }^{(14)}$; c) Ditado ortográfico ${ }^{(15)}$;) Avaliação de fluência da escrita ${ }^{(7)}$.

\section{Análise dos dados}

Para investigação da estrutura dimensional da ELE, foi utilizada a análise fatorial exploratória. Nesta análise, a matriz de variância/covariância dos dados provenientes da escala foi submetida ao método de estimação robust weighted least squares a fim de: 1) obter-se resultados representativos da população geral, ou seja, é possível extrapolar os dados da amostra para a população; 2) modelar casos omissos (missing values) e dados provenientes de escala ordinal, já que os estimadores usuais como o método de máxima verossimilhança pressupõem itens ou variáveis de nível intervalar e distribuição normal desses indicadores ${ }^{(16)}$. No caso de escalas de escalas do tipo Likert, os itens são de variáveis de nível ordinal e, para contemplar a natureza deste tipo de variável, análises fatoriais com o uso de estimadores do tipo RWLS tendem a acertar com mais frequência o número de fatores subjacentes aos dados, produzindo ainda estimativas paramétricas mais consistentes de cargas fatoriais e correlações entre fatores ${ }^{(17)}$. Os índices de ajuste (valores de referência) considerados para a adequação dos modelos foram: Comparative Fit Index (CFI > 0,90); Tucker-Lewis index (TLI $>0,90$ ); Standardized Root Mean Residual (SRMR) e RMSEA (Root Mean Square Error of Aproximation), todos com valores ideais próximos ou inferiores a 0,08 . Outros índices como Akaike Information Criterion (AIC) e Bayesian Information Criterion (BIC) foram utilizados na comparação entre diferentes hipóteses de modelos teóricos (valores menores indicando melhor adequação). A fidedignidade de cada fator foi estimada por meio do coeficiente Alfa de Cronbach, sendo considerados adequados valores maiores que $0,6^{(18)}$.

A fim de avaliar as propriedades psicométricas de cada item da ELE pela teoria de resposta ao item (TRI), o modelo

Tabela 1. Dados sócio-demográficos dos professores

\begin{tabular}{|c|c|c|c|c|c|c|c|c|}
\hline \multicolumn{3}{|c|}{ Idade (anos) } & \multicolumn{3}{|c|}{ Tempo profissional (anos) } & \multicolumn{3}{|c|}{ Escolaridade (\%) } \\
\hline Méd. (DP) & Máx. & Mín. & Méd. (DP) & Máx. & Mín. & Grad. Incomp. & Grad. Comp. & Pós-Grad. \\
\hline $41,89(8,44)$ & 56 & 27 & $9,62(7,36)$ & 20 & 2 & 8,3 & 41,6 & 50 \\
\hline
\end{tabular}


de créditos parciais de Masters ${ }^{(19)}$ (1978, extensão para itens politômicos do modelo dicotômicos de Rasch [1960]) foi empregado. O modelo de créditos parciais estima de forma conjunta o nível de habilidade dos indivíduos e a dificuldade dos itens em que ambos os parâmetros são representados em um mesmo contínuo linear simples, em unidades de logaritmos de chance (logits, log-odds unit), em que os itens e as estimativas do traço latente se encontram em uma mesma métrica, portanto a estimativa da habilidade representará uma probabilidade de resposta ou endosso da categoria do item. A investigação dos seus pressupostos foram testados por meio da: 1) análise da unidimensionalidade deste instrumento, obtida através de análise fatorial; 2) monotonicidade (princípio em que a probabilidade de endossar uma determinada categoria de um item aumenta de acordo com o aumento da variável latente do participante); e 3) independência local (os itens do teste não dependem um do outro ou não influenciam um ao outro para obtenção da resposta).

Pelo TRI também foi mensurado os resíduos infit e outfit dos itens. Neste estudo, foram considerados valores de 0,5 a 1,5, uma vez que de acordo com Wright e Linacre ${ }^{(20)}$ estas faixas do infit e do outfit fornecem parâmetros produtivos de mensuração. Como critério de desempate das duas medidas de adequação, a significância do teste qui-quadrado foi utilizada. A exemplo, se algum item apresentou infit insatisfatório e outfit satisfatório, a comparação da diferença entre os valores previstos de cada item pelo modelo com os valores empíricos coletados foi considerada por meio do teste qui-quadrado.

Para a analisar a validade convergente, correlações de Spearman foram calculadas. Por fim, a análise de estabilidade temporal foi realizada por meio de teste e reteste das respostas dos professores à escala, com intervalo de dois a quatro meses. Para que um instrumento seja confiável e estável temporalmente as correlações devem se situar entre 0,8 ou mais ${ }^{(12)}$. Foram utilizados Statistical Package for the Social Sciences, Mplus ${ }^{(21)}$ e o $R^{(22)}$, além das funções implementadas pelos pacotes $p s y c h^{(23)}$ e $\operatorname{mirt}^{(24)}$ para análise.

\section{RESULTADOS}

Como são muitas análises distintas (análise fatorial, TRI, validade convergente; Teste-reteste), os resultados são apresentados em diferentes blocos.

\section{Análise fatorial: ELE tem um fator}

A análise fatorial indicou que apenas um fator foi extraído da escala. (RLWS: $\chi^{2}=-6150,32 ; \mathrm{N}$ par $=16 ; \mathrm{CFI}=0,98$; $\mathrm{TLI}=0,98 ; \mathrm{RMSEA}=0,12 ; \mathrm{SRMR}=0,05$ ). Análises adicionais (scree plot; teste de Cattell( ${ }^{(25)}$ ); análises paralelas de Horn ${ }^{(26)}$ também reforçaram a existência de um fator. A matriz fatorial indica excelentes cargas fatoriais (entre 0,72 à 0,96 ) e de covariância dos itens (Tabela Suplementar 01 do material suplementar).

A análise da Consistência interna (Tabela Suplementar 02 do material suplementar) mostrou valores elevados (coeficiente alpha de Cronbach $=0,968)$, indicando um alto grau da covariância entre os itens da escala (o alpha menor foi 0,9643 e o maior, 0,9678).

\section{Teoria de Resposta ao Item (TRI): evidências que independem da amostra}

O resultado da TRI (Tabela 2) mostra que os itens foram monotônicos, ou seja, a variabilidade estaria atrelada a um único construto (leitura e escrita; valores iguais ou acima de 0,6). Os valores limítrofes do item $15(0,59)$ não foi considerado, uma vez que os índices dos ajustes (Infit e Outfit) foram bons. Os itens 1 e 10 na análise de resíduos apontou desajustes limítrofes nos valores de Infit e Outfit dos itens 1

Tabela 2. Análise de resíduos do modelo de créditos parciais utilizando quatro categorias de resposta

\begin{tabular}{|c|c|c|c|c|c|c|c|c|}
\hline Item & Outfit & Infit & Monotonicidade & p.S_X2 & $\begin{array}{c}\text { Nunca - } \\
\text { Raramente }\end{array}$ & $\begin{array}{c}\text { Raramente - } \\
\text { Âs Vezes }\end{array}$ & $\begin{array}{l}\text { Às vezes - } \\
\text { Frequentemente } \\
\text { / Sempre }\end{array}$ & Dificuldade \\
\hline Item.1 & 0,48 & 0,53 & 0,78 & 0,04 & 0,02 & 0,66 & 1,14 & 0,60 \\
\hline Item.2 & 0,41 & 0,57 & 0,77 & 0,08 & 0,15 & 0,65 & 0,79 & 0,52 \\
\hline Item.3 & 1,23 & 1,18 & 0,71 & 0,56 & 0,31 & 0,54 & 2,16 & 1,00 \\
\hline Item.4 & 0,57 & 0,72 & 0,75 & 0,65 & $-0,07$ & 0,99 & 2,04 & 0,98 \\
\hline Item.5 & 0,67 & 0,80 & 0,72 & 0,32 & $-0,35$ & 0,89 & 1,69 & 0,75 \\
\hline Item.6 & 0,53 & 0,75 & 0,77 & 0,05 & 0,85 & 2,42 & 2,40 & 1,89 \\
\hline Item.7 & 0,51 & 0,60 & 0,77 & 0,37 & $-0,44$ & 0,63 & 1,80 & 0,66 \\
\hline Item.8 & 1,15 & 1,16 & 0,68 & 0,17 & $-0,89$ & 0,53 & 0,88 & 0,17 \\
\hline Item.9 & 0,63 & 0,68 & 0,77 & 0,29 & $-1,58$ & $-0,06$ & 0,89 & $-0,25$ \\
\hline Item.10 & 1,64 & 1,28 & 0,62 & 0,04 & $-0,80$ & $-0,56$ & 0,97 & $-0,13$ \\
\hline Item.11 & 1,18 & 1,28 & 0,67 & 0,26 & $-0,90$ & 0,63 & $-0,12$ & $-0,13$ \\
\hline Item.12 & 1,80 & 1,28 & 0,60 & 0,16 & $-0,01$ & 1,21 & 1,26 & 0,82 \\
\hline Item.13 & 0,66 & 0,90 & 0,71 & 0,02 & $-0,07$ & 0,61 & 1,40 & 0,65 \\
\hline Item.14 & 0,86 & 0,98 & 0,72 & 0,03 & $-0,97$ & 0,26 & 1,95 & 0,41 \\
\hline Item.15 & 1,19 & 1,46 & 0,59 & 0,47 & $-0,76$ & 1,53 & 2,52 & 1,10 \\
\hline Item.16 & 0,77 & 0,87 & 0,69 & 0,11 & $-0,43$ & 0,88 & 1,83 & 0,76 \\
\hline
\end{tabular}

Legenda: p.S_X2 = significância. Fonte em negrito indicam desajuste no valor dos outfit e infit 
Tabela 3. Normas de interpretação e distribuição da amostra

\begin{tabular}{|c|c|c|}
\hline \multicolumn{3}{|c|}{ Distribuição Escores por Percentil } \\
\hline Percentil & Escores Brutos & Escores TRI \\
\hline $0 \%$ & 16 & $-3,747$ \\
\hline $5 \%$ & 16 & $-3,747$ \\
\hline $10 \%$ & 16 & $-3,747$ \\
\hline $15 \%$ & 19 & $-2,069$ \\
\hline $20 \%$ & 20,6 & $-1,585$ \\
\hline $25 \%$ & 24 & $-0,999$ \\
\hline $30 \%$ & 26,9 & $-0,604$ \\
\hline $35 \%$ & 27,55 & $-0,502$ \\
\hline $40 \%$ & 31 & $-0,189$ \\
\hline $45 \%$ & 32 & $-0,098$ \\
\hline $50 \%$ & 33,5 & 0,074 \\
\hline $55 \%$ & 36 & 0,236 \\
\hline $60 \%$ & 36 & 0,313 \\
\hline $65 \%$ & 39,9 & 0,606 \\
\hline $70 \%$ & 44,1 & 0,937 \\
\hline $75 \%$ & 47 & 1,118 \\
\hline $80 \%$ & 51 & 1,431 \\
\hline $85 \%$ & 54,05 & 1,811 \\
\hline $90 \%$ & 58 & 2,392 \\
\hline $95 \%$ & 61,7 & 3,311 \\
\hline $100 \%$ & 64 & 4,942 \\
\hline
\end{tabular}

Legenda: Escores brutos: total do escore da ELE; Escores TRI: nível de habilidade das crianças; Percentil: $0 \%=$ nenhuma dificuldade; $100 \%$ = máxima dificuldade $\left(\right.$ Outfit $=0,48 ;$ Infit $\left.=0,53 ; x^{2}=0,04\right)$ e $10($ Outfit $=1,64 ;$ Infit $=$ 1,$\left.28 ; x^{2}=0,04\right)$. Ademais, observou-se que os thresholds do item 11 (Nunca-raramente $=-0,90$; raramente-às vezes $=0,63$; às vezes-frequentementelsempre $=-0,12$ ) não apresentaram uma relação adequada entre a frequência de manifestação do sintoma e a habilidade calculada pela TRI.

A escala consegue mensurar a habilidade das crianças que se encontram a partir do percentil 10\% (Tabela 3). Sobrepondo os parâmetros de dificuldade (Tabela 2) e a curva de habilidade (Figura 1 - material suplementar), pode-se observar (Tabela 3) que a parte mais informativa da escala fica entre o percentil $10 \%$ e $90 \%$, entre os escores 16 e 58 .

\section{Validade convergente: a relação entre a ELE e os testes de leitura e escrita}

A correlação de Spearman foi moderada $(\geq 0,40$ a $<0,60)$ para 11 das 17 variáveis externas (Tabela 4).

\section{Teste-reteste: a estabilidade da avaliação feita com a ELE depois de um intervalo de tempo}

A avaliação da estabilidade temporal foi realizada pelas correlações das estimativas de traço latente da primeira com a segunda coleta de dados $\left(r_{s}=0,80, p<0,0001\right)$. Não houve diferenças significativas entre as médias das estimativas nos dois momentos (diferença média $=-0,001, t(29)=-0,025324, p=0,98$ ).

Tabela 4. Correlações entre os resultados da ELE e das tarefas de leitura e escrita

\begin{tabular}{|c|c|c|c|c|c|c|c|c|}
\hline & & Escola 1 & Escola 2 & Escola 3 & Escola 4 & Escola 5 & Escola 6 & Geral \\
\hline Ditado & CFG & 0,44 & 0,74 & 0,63 & 0,33 & 0,85 & 0,84 & 0,63 \\
\hline Balanceado - Erros & $\mathrm{RCS}$ & 0,59 & 0,46 & 0,74 & 0,24 & 0,84 & 0,84 & 0,70 \\
\hline \multirow[t]{3}{*}{ Média (DP) } & $\mathrm{RCC}$ & 0,50 & 0,69 & 0,23 & 0,34 & $0,008^{*}$ & 0,58 & 0,30 \\
\hline & IR & 0,01 & 0,68 & 0,34 & 0,35 & 0,84 & 0,60 & 0,44 \\
\hline & Total & 0,37 & 0,71 & 0,62 & 0,34 & 0,86 & 0,91 & 0,65 \\
\hline Cópia Letras & Total & $0,007^{*}$ & $-0,66$ & $-0,29$ & $-0,01^{*}$ & $-0,91$ & $-0,63$ & $-0,44$ \\
\hline \multirow[t]{2}{*}{ Média (DP) } & Erros & 0,43 & $-0,11^{\star}$ & 0,24 & $0,02^{*}$ & $-0,46$ & $-0,18^{\star}$ & $0,015^{\star}$ \\
\hline & Velocidade & $0,007^{*}$ & $-0,66$ & $-0,29$ & $-0,01^{*}$ & $-0,91$ & $-0,63$ & $-0,41$ \\
\hline $\begin{array}{c}\text { Compreensão de } \\
\text { Textos }\end{array}$ & Espontânea & 0,22 & $-0,55$ & $-0,35$ & $-0,85$ & $0,03^{\star}$ & $-0,72$ & $-0,38$ \\
\hline \multirow[t]{3}{*}{ Média (DP) } & Dirigida & 0,18 & $-0,48$ & $-0,19$ & $-0,84$ & $-0,35$ & $-0,68$ & $-0,32$ \\
\hline & $\begin{array}{c}\text { Tempo (s) por } \\
\text { c, dirigida }\end{array}$ & $-0,34$ & 0,57 & 0,31 & 0,68 & 0,69 & 0,84 & 0,43 \\
\hline & Velocidade & 0,18 & $-0,73$ & $-0,17$ & $-0,48$ & 0,87 & $-0,61$ & $-0,29$ \\
\hline Leitura de Palavras e & Total & 0,52 & 0,53 & 0,31 & 0,46 & 0,79 & 0,82 & 0,52 \\
\hline Pseudopalavras & Palavras & 0,41 & 0,74 & 0,38 & 0,56 & 0,55 & 0,85 & 0,50 \\
\hline \multirow[t]{3}{*}{ Média (DP) } & Pseudo & 0,55 & 0,27 & 0,21 & 0,16 & 0,66 & 0,53 & 0,34 \\
\hline & $\begin{array}{c}\text { Erros por } \\
\text { velocidade }\end{array}$ & 0,37 & 0,65 & 0,40 & 0,61 & 0,99 & 0,88 & 0,58 \\
\hline & Velocidade & 0,19 & $-0,89$ & $-0,38$ & $-0,50$ & $-0,83$ & $-0,82$ & $-0,56$ \\
\hline
\end{tabular}

Legenda: Correlações com * não foram significativas. 1) Tipos de erros no Ditado Balanceado: a) Conversor fonema-grafema (CFG); b) Regras contextuais simples (RCS); Regras contextuais complexas (RCC); Irregularidade da Língua (IR); Total de erros na tarefa (Total), 2) Cópia de caracteres: a) Total de letras copiadas (Total); b) Total de erros ortográficos (Erros); Velocidade de caracteres por minuto (Velocidade); 3) Compreensão de textos: a) Número de proposições evocadas espontaneamente de um total de 18 (Espontânea); b) Número de acerto as questões formuladas sobre o texto com pontuação máxima de 10 pontos (Dirigida); c) Coeficiente entre o tempo total, em segundos, e o número de acerto as questões dirigidas (Tempo (s) por c, dirigida); d) Velocidade de palavras lidas por minuto (Velocidade), 4) Leitura de Palavras e Pseudopalavras: a) Total de erros na tarefa (Total); b) Total de erros em palavras (Palavras); c) Total de erros em pseudopalavras (Pseudopalavras); d) coeficiente do total de erros na tarefa pela velocidade (Erros por velocidade); e) Velocidade de palavras lidas por minuto (Velocidade) 


\section{DISCUSSÃO}

O resultado da análise fatorial indica que a escala avalia um único construto, ou seja, as questões de leitura e escrita, medidos nesta testagem, se comportaram como uma única habilidade. Desta maneira, evidencia-se que diferentes habilidades cognitivas como funções executivas e atenção, por exemplo, não estão interferindo no resultado da escala.

Sabe-se que leitura e escrita envolverem processos cognitivos com diferentes peculiaridades ${ }^{(27)}$, podendo inclusive serem aprendidas separadamente. Devido a isso, acreditava-se que a ELE fosse composta de dois construtos de habilidade. Entretanto, por serem habilidades muito interdependentes ${ }^{(15)}$, os itens da escala não conseguiram mensurar suas peculiaridades, pelo menos em crianças do terceiro ano.

Os resultados da análise da estrutura interna possibilitou obtenção de um indicativo de fidedignidade da escala e dos sintomas investigados ${ }^{(11)}$. No entanto, vale ressaltar que valores tão elevados do Alpha de Cronbach pode representar uma redundância dos itens, ou seja, a presença de itens muito semelhantes na escala. Para investigar essa questão realizou-se as análises da TRI uma vez que esta análise indica o nível de habilidade que determinado item avalia ${ }^{(11,12)}$, permitindo evitar a sobreposição dos itens.

Primeiramente, os resultados da TRI confirmaram que a ELE avalia um único construto (valores de monotonicidade iguais ou superiores a 0,6 ). Os resultados da análise de resíduos indicaram que os itens 1 (Demora mais tempo que os colegas para ler palavras) e 10 (Conta oralmente uma história melhor do que consegue escrevê-la) apresentaram desajustes nos valores de outfit e infit. No entanto, pelo valor clínico das questões e por se considerar que os desajustes eram limítrofes, ambos foram mantidos.

Na análise da frequência dos sintomas (Nunca à Raramente; Raramente à Às Vezes; Às Vezes à Frequentemente/sempre), houve discrepância entre a frequência de manifestação do sintoma e a habilidade calculada pela TRI no item 11 (Demora mais tempo que os colegas nos momentos de cópia (como por exemplo do quadro)). Isso pode estar indicando que mais de uma variável interfere no processo de resposta deste item ${ }^{(28)}$, como por exemplo o fator atencional. Não se compreendeu necessária sua exclusão, uma vez que todas as outras medidas advindas do item apresentaram bons valores.

Mais uma medida de fidedignidade da escala foi obtida pela análise de TRI. Observando-se a sobreposição dos parâmetros de dificuldade dos itens (Tabela 2) aos da curva de informação (Figura 1 - material suplementar), pode-se verificar que a avaliação de crianças é mais precisa nas faixas de habilidade "média-alta", "média" e "média-baixa". A análise também mostrou que não houve superavaliação e pouca subavaliação das faixas de habilidade mensuradas pela ELE.

$\mathrm{Na}$ Tabela 3, os percentis, iguais ou inferiores a $10 \%$, bem como, os iguais ou superiores a $90 \%$ não se encontram na faixa mais informativa da curva de informação da TRI (Figura 1 - material suplementar), sendo assim, o nível de habilidade das crianças dessas faixas é mal avaliado. Destaca-se que a escala não é capaz de apontar as diferenças de habilidade até o percentil 10, onde o valor da TRI é de $-3,747$. Este resultado sugere que até certo grau de habilidade todos os desempenhos dos alunos sejam classificados na categoria "Nunca". Também, sugere-se que alunos $(\mathrm{n}=9)$ que obtiverem pontuações iguais ou superiores à 58 pontos (percentil 90) devam ser encaminhados para uma avaliação diagnóstica devido ao grau de dificuldade que apresentam. Estas crianças estariam em risco para a DD; a porcentagem (aproximadamente 9\% da amostra), corrobora a prevalência de $5-10 \%$ de DD relatada em diferentes países ${ }^{(29)}$.

No que concerne a validade convergente, 11 das 17 variáveis apresentaram correlação moderada. Na literatura, não há consenso sobre a definição de intensidade da correlação adequada para uma validade convergente ${ }^{(30)}$. Urbina ${ }^{(11)}$ aponta que a correlação deve apresentar valores fortes, enquanto, DeVon et al. ${ }^{(30)} \mathrm{e}$ colaboradores, em sua revisão, indicam que valores superiores 0,50 são pouco frequentes, pois, muitas vezes, não é possível dispor de uma tarefa com as mesmas especificidades e validada para realizar a correlação.

Em relação aos testes empregados, há um problema sobre o critério de comparação para o presente estudo. No Brasil, não existe um padrão-ouro para a avaliação de leitura e escrita. O mais difundido instrumento de avaliação, citado em 478 estudos (Google Acadêmico, 2016) é o Teste de Desempenho Escolar$\mathrm{TDE}^{(31)}$. Entretanto, a versão disponível na época da pesquisa havia sido construída há mais de vinte anos, encontrando-se desatualizada ${ }^{(32)}$. Nenhum dos testes utilizados neste estudo apresentam estudos de validação e apenas um (Ditado Balanceado) apresenta normatização ${ }^{(15)}$.

Com base nos argumentos de DeVon et al. ${ }^{(30)}$, compreende que o resultado da ELE tem importante relação com o desempenho nas tarefas de leitura e escrita. A validade convergente precisou ser realizada por escola, pois houve importantes diferenças nas médias de desempenhos nas tarefas de leitura e escrita ${ }^{(7)}$ entre instituições. Com esta variação, professoras de diferentes instituições ao avaliarem o item de frequência de erros ortográficos na escrita de um aluno que apresentou 60 erros no Ditado Balanceado ${ }^{(15)}$, provavelmente pontuariam a ELE para este aluno de forma distinta.

As diferenças encontradas nas médias de desempenho nas provas de leitura e escrita desta pesquisa podem, em boa parte, estar relacionadas à variação do método e do currículo destas escolas. As Diretrizes Curriculares Nacionais da Educação Básica $^{(33)}$ limitam-se a apontar princípios metodológicos (interdisciplinaridade e problematização), sem especificar quais são e como devem ser trabalhados. Fica, assim, a cargo do professor escolher a melhor metodologia de ensino para seu grupo, de forma que as estratégias de apresentação do conteúdo escolar podem variar de educador para educador, levando assim a diferença de desempenho dos alunos.

A ELE investiga leitura e escrita, habilidades que envolvem diferentes processos cognitivos, mas que são interdependentes, podendo assim, ser comparada a uma bateria de testes. Por exemplo, todas as tarefas de compreensão estão, essencialmente, relacionadas apenas ao item 7 da escala, e as relativas ao Ditado Balanceado apresentam uma relação mais intrínseca com o item 8. Apesar da análise fatorial ter indicado que o desempenho em leitura e escrita possui uma forte correlação 
entre si, podendo ser consideradas representantes de um único fator, esta maior especificidade das tarefas pode ter diminuído a força de correlação, como se observa ao comparar baterias de teste a uma tarefa isolada ${ }^{(11)}$.

Uma única variável não apresentou correlação com a escala, que foi o número de erros cometidos na cópia. Pode-se supor que isso deva ter ocorrido uma vez que as crianças com dificuldade persistente, pela consciência de seu problema, criam estratégias para revisar sua produção, a fim de evitar o erro. Desta maneira, a precisão não é impactada, mas o que diminui é a velocidade com que fazem.

A correlação mais fraca foi a da velocidade de leitura. $\mathrm{Na}$ prática escolar, não há parâmetros de fluência leitora( ${ }^{(34)}$, portanto esta avaliação é de ordem subjetiva. A baixa correlação com os escores de compreensão pode ser reflexo de falhas conceituais sobre compreensão e do instrumento utilizado. Neste sentido, estudos indicam lacunas no conhecimento do professor frente aos processos que são subjacentes ao desenvolvimento da leitura ${ }^{(35)}$.

A subjetividade da percepção dos professores sobre diferenças entre alunos também pode estar relacionada à força das correlações encontradas. Percebe-se que a intensidade da correlação variou muito entre as instituições, variando, inclusive, de positivo para negativo. A formação do professor e o seu tempo de atuação podem estar diretamente implicados nesta diferença entre instituições, bem como outras variáveis sociais e demográficas das escolas.

Por fim, sobre o valor do coeficiente de estabilidade temporal (teste e reteste), interpreta-se que valores ótimos são aqueles que se situam iguais ou superiores a $0,90^{(11)}$, entretanto, valores até 0,80 são considerados aceitáveis ${ }^{(12)}$. Diversos fatores podem explicar a obtenção de um valor inferior ao mais desejado.

Destacam-se que questões como o intervalo de tempo entre as avaliações e a possível diminuição na motivação do participante ao responder novamente a um teste interferem no valor da correlação ${ }^{(12)}$. É indicado um intervalo de 15 a 30 dias entre as duas medidas. As devoluções desta etapa de reavaliação só foram realizadas no período entre dois a quatro meses da avaliação inicial; entre os motivos, houve atraso na entrega, pois inicialmente apenas uma professora havia aceitado participar, o que também parece apontar para uma diminuição da motivação da amostra de professores.

\section{CONCLUSÃO}

Os processos apresentados permitiram a obtenção de uma série de evidências de validade, segundo os princípios da American Psychological Association apontados pelo Conselho de Psicologia ${ }^{(36)}$, da escala de rastreio ELE (Anexo A). Apesar da ELE ter sido construída para avaliar as habilidades de leitura e escrita de escolares do $1^{\circ}$ ao $5^{\circ}$ ano, sua validade ficou restrita, por este trabalho, à alunos de $3^{\circ}$ ano.

Como dito na introdução, o marco de escolha para realizar os primeiros estudos ser o $3^{\circ}$ ano devia-se aos critérios diagnósticos exigirem a exclusão de falhas metodológicas de ensino e as concepções, à época, do PNAIC e atualmente da BNCC. No entanto, em 2019, mudanças conceituais levaram a alterações importantes na atual Política Nacional de Alfabetização ${ }^{(37)}$. A nova concepção almeja que ao final do segundo ano do ensino fundamental, as crianças já sejam hábeis em ler e escrever textos simples. Essa nova concepção não invalida o estudo atual e muito menos a escala, sobremaneira. Os seus itens continuam representando os sintomas do transtorno, a mudança não implicará alterações nas análises estatísticas, principalmente, as que se referem aos itens e seus resultados se mostraram correlacionados ao desempenho de provas de leitura e escrita. Ademais, a BNCC, como discutido anteriormente, continua a manter o $3^{\circ}$ ano como marco da alfabetização e é no $3^{\circ}$ ano que se faz a avaliação nacional de alfabetização.

Este trabalho possibilitou a produção de uma ferramenta, que avalia o que se propõem a avaliar, para suprir uma lacuna na área. Através da ELE, médicos, fonoaudiólogos, psicólogos e professores terão indícios confiáveis para uma possível identificação do aluno com risco de DD. A ELE poderá também ser utilizada por pesquisadores que desejarem selecionar crianças, neste momento de $3^{\circ}$ ano, com e sem prejuízos no desenvolvimento das habilidades de leitura/escrita. A escala pode servir de instrumento de rastreamento populacional para pesquisa.

\section{ACKNOWLEDGEMENTS}

A Coordenação de Aperfeiçoamento de Pessoal de Nível Superior (CAPES), Observatório da Educação - Projeto ACERTA (processo 23038.002530/2013-93) que financiou este projeto. Agradecemos também aos Juízes especialistas que avaliaram a escala e ao Me. Euclides José de Mendonça Filho pela sua contribuição com as análises estatísticas.

\section{REFERENCES}

1. APA: American Psychiatric Association. DSM-5: manual diagnóstico e estatístico de transtornos mentais. Porto Alegre: Artmed; 2014.

2. IBGE: Instituto Brasileiro de Geografia e Estatística. Taxa de analfabetismo das pessoas de 15 anos ou mais de idade, por sexo - Brasil - 2007/2014 [Internet]. Rio de Janeiro: IBGE; 2016 [citado em 2016 Jul 27]. Disponível em: http://brasilemsintese.ibge.gov.br/educacao/taxa-de-analfabetismodas-pessoas-de-15-anos-ou-mais.html

3. Costa AC, Toazza R, Bassoa A, Portuguez MW, Buchweitz A. Ambulatório de Aprendizagem do Projeto ACERTA (Avaliação de Crianças Em Risco de Transtorno de Aprendizagem): métodos e resultados em dois anos. In: Salles J, Haase VG, Malloy-Diniz LF, editores. Neuropsicologia do desenvolvimento: infância e adolescência. Porto Alegre: Artmed; 2015. p. 151-8.

4. Colenbrander D, Ricketts J, Breadmore HL. Early identification of dyslexia understanding the issues. Lang Speech Hear Serv Sch. 2018;49(4):817-28. http://dx.doi.org/10.1044/2018_LSHSS-DYSLC-18-0007. PMid:30458543.

5. Torres D, Ciasca S. Correlação entre a queixa do professor e a avaliação psicológica em crianças de primeira série com dificuldades de aprendizagem. Rev Psicopedag. 2007;24(73):18-29.

6. Bussing R, Fernandez M, Harwood M, Wei Hou, Garvan CW, Eyberg SM, et al. Parent and teacher SNAP-IV ratings of attention deficit hyperactivity disorder symptoms: psychometric properties and normative ratings from a school district sample. Assessment. 2008;15(3):317-28. http://dx.doi. org/10.1177/1073191107313888. PMid:18310593.

7. Bassôa A. Construção e evidências de fidedignidade e validade de uma Escala de Leitura e Escrita (ELE) para o rastreio de crianças com dificuldades escolares [dissertação]. Porto Alegre: Pontifícia Universidade Católica do Rio Grande do Sul; 2016. 
8. Torgesen JK. The prevention of reading difficulties. J Sch Psychol. 2002;40(1):7-26. http://dx.doi.org/10.1016/S0022-4405(01)00092-9.

9. Torgesen JK. Individual differences in response to early interventions in reading: the lingering problem of treatment resisters. Learn Disabil Res Pract. 2000;15(1):55-64. http://dx.doi.org/10.1207/SLDRP1501_6.

10. Gresham FM, Elliott SN. Inventário habilidades sociais, problemas comportamento e competência acadêmica para crianças - SSRS. São Paulo: Casa do Psicólogo; 2016.

11. Urbina S. Essentials of psychological testing. 2nd ed. New Jersey: Wiley; 2014.

12. Pasquali L. Psicometria: teoria dos testes na psicologia e na educação. 5. ed. Rio de Janeiro: Vozes; 2013.

13. Salles JF. Habilidades e dificuldades de leitura e escrita em crianças de $2^{\text {a }}$ série: abordagem neuropsicológica cognitiva [dissertação]. Porto Alegre: Universidade do Estado do Rio Grande do Sul; 2005.

14. Saraiva RA, Moojen SMP, Munarski R. Avaliação da compreensão leitora de textos expositivos para fonoaudiólogos e psicopedagogos. São Paulo: Casa do Psicólogo; 2005.

15. Moojen SMP. A escrita ortográfica na escola e na clínica: teoria, avaliação e tratamento. São Paulo: Casa do Psicólogo; 2011.

16. Brown TA. Confirmatory factor analysis for applied research. 2nd ed. USA: Guilford Publications; 2015.

17. Holgado-Tello FP, Chacón-Moscoso S, Barbero-García I, Vila-Abad E. Polychoric versus Pearson correlations in exploratory and confirmatory factor analysis of ordinal variables. Qual Quant. 2009;44(1):153-66. http:// dx.doi.org/10.1007/s11135-008-9190-y.

18. Hair JF, Black WC, Anderson R, Babin B. Multivariate data analysis: a global perspective. 7th ed. Upper Saddle River: Pearson Education; 2010.

19. Masters GN. A Rasch model for partial credit scoring. Psychometrika. 1982;47(2):149-74. http://dx.doi.org/10.1007/BF02296272.

20. Wright BD, Linacre JM. Reasonable mean-square fit values. Rasch Meas Trans. 1994;8:370.

21. Muthén LK, Muthén B. Mplus user's guide. 6th ed. Los Angeles, CA: Muthén \& Muthén; 2010.

22. R Core Team. R: a language and environment for statistical computing [Internet]. Vienna: Foundation for Statistical Computing; 2015 [citado em 2016 Ago 27]. Disponível em: https://cran.r-project.org

23. Revelle W. Psych: procedures for personality and psychological research [Internet]. Evanston, Illinois: Northwestern University; 2015 [citado em 2016 Ago 27]. Disponível em: http://cran.r-project.org/package=psych

24. Chalmers RP. mirt: a multidimensional item response theory package for the R environment. J Stat Softw. 2012;48(6):1-29. http://dx.doi.org/10.18637/ jss.v048.i06.
25. Cattell RB. The scree test for the number of factors. Multivariate Behav Res. 1966;1(2):245-76. http://dx.doi.org/10.1207/s15327906mbr0102_10. PMid:26828106.

26. Horn JL. A rationale and test for the number of factors in factor analysis. Psychometrika. 1965;30(2):179-85. http://dx.doi.org/10.1007/BF02289447. PMid:14306381.

27. Graham S, Hebert M. Writing to read: A meta-analysis of the impact of writing and writing instruction on reading. Harv Educ Rev. 2011;81(4):71044. http://dx.doi.org/10.17763/haer.81.4.t2k0m13756113566.

28. DeMars C. Item response theory. USA: Oxford University Press; 2010. http://dx.doi.org/10.1093/acprof:oso/9780195377033.001.0001.

29. Law JM, Vandermosten M, Ghesquiere P, Wouters J. The relationship of phonological ability, speech perception, and auditory perception in adults with dyslexia. Front Hum Neurosci. 2014;8(482):482. http://dx.doi. org/10.3389/fnhum.2014.00482. PMid:25071512.

30. DeVon HA, Block ME, Moyle-Wright P, Ernst DM, Hayden SJ, Lazzara DJ, et al. A psychometric toolbox for testing validity and reliability. J Nurs Scholarsh. 2007;39(2):155-64. http://dx.doi.org/10.1111/j.15475069.2007.00161.x. PMid:17535316.

31. Stein LM. TDE: teste de desempenho escolar: manual para aplicação e interpretação. São Paulo: Casa do Psicólogo; 1994. 17 p.

32. Athayde MDL, Mendonça EJD Fo, Fonseca RP, Stein LM, Giacomoni CH. Desenvolvimento do subteste de leitura do Teste de Desempenho Escolar II. Psico-USF. 2019;24(2):245-57. http://dx.doi.org/10.1590/141382712019240203.

33. Brasil. Ministério da Educação. Diretrizes Curriculares Nacionais da Educação Básica. Brasília: MEC, SEB, DICEI; 2013.

34. Komeno EM, Ávila CRBD, Cintra IDP, Schoen TH. Velocidade de leitura e desempenho escolar na última série do ensino fundamental. Estud Psicol. 2015;32(3):437-47. http://dx.doi.org/10.1590/0103-166X2015000300009.

35. Oliveira FJD, Silveira MIM. A compreensão leitora e o processo inferencial em turmas do nono ano do ensino fundamental. Revista da FAEEBA. 2014;23(41):91-104. http://dx.doi.org/10.21879/faeeba2358-0194.v23. $\mathrm{n} 41.826$

36. Conselho Federal de Psicologia. Avaliação psicológica: diretrizes na regulamentação da profisssão. 1. ed. Brasília: CFP; 2010.

37. Brasil. Ministério da Educação. Secretaria de Alfabetização. Política Nacional de Alfabetização (PNA). Brasília: MEC, SEALF; 2019.

\section{Contribuição dos autores}

ABS participou de todas as etapas da pesquisa, desde a construção do protocolo, as coletas, análises estatísticas e escrita do paper. ABZ supervisionou toda a pesquisa, ajudou nas análises e na escrita do artigo. ACC e RT trabalharam na construção da escala e na revisão do manuscrito. 


\section{ANEXO A. ESCALA DE RASTREIO}

Nome do aluno:

ESCALA DE LEITURA E ESCARITA - ELE VERSÃO PROFESSORES

A lista abaixo descreve algumas dificuldades específicas que podem aparecer quando a criança/adolescente apresenta prejuízo na aprendizagem da leitura e/ou da escrita. Para cada frase, marque um " $X$ " na frequência que corresponde à resposta que melhor

\begin{tabular}{|c|c|c|c|c|}
\hline & Nunca & Raramente & Às vezes & $\begin{array}{c}\text { Frequentemente/ } \\
\text { Sempre }\end{array}$ \\
\hline \multicolumn{5}{|l|}{ 1. Demora mais tempo que os colegas para ler palavras; } \\
\hline \multicolumn{5}{|l|}{ 2. Demora mais tempo que os colegas para ler textos; } \\
\hline \multicolumn{5}{|l|}{ 3. Troca letras ao ler sílabas e palavras na leitura oral; } \\
\hline \multicolumn{5}{|l|}{$\begin{array}{l}\text { 4. Fica ensaiando a palavra (lendo em voz baixa) antes de ler } \\
\text { oralmente; }\end{array}$} \\
\hline \multicolumn{5}{|l|}{$\begin{array}{l}\text { 5. Gagueja, treme, fica vermelho e/ou rele algumas palavras } \\
\text { na hora de ler oralmente; }\end{array}$} \\
\hline \multicolumn{5}{|l|}{$\begin{array}{l}\text { 6. Inventa", "chuta", troca a palavra por outra parecida ou } \\
\text { parece tentar adivinhar as palavras na leitura; }\end{array}$} \\
\hline \multicolumn{5}{|l|}{$\begin{array}{l}\text { 7. Não compreende o que lê, (p,ex,: após ler o texto } \\
\text { não entende o que é para fazer e/ou não entende o que } \\
\text { aconteceu com os personagens); }\end{array}$} \\
\hline \multicolumn{5}{|l|}{ 8. Troca, "come"/omite ou acrescenta letras na escrita; } \\
\hline \multicolumn{5}{|l|}{$\begin{array}{l}\text { 9. Escreve textos muito simples, pobres em ideias e detalhes } \\
\text { se comparado aos colegas; }\end{array}$} \\
\hline \multicolumn{5}{|l|}{$\begin{array}{l}\text { 10. Conta, oralmente, uma história melhor do que consegue } \\
\text { escrevê-la; }\end{array}$} \\
\hline \multicolumn{5}{|l|}{$\begin{array}{l}\text { 11. Demora mais tempo que os colegas nos momentos de } \\
\text { cópia (como por exemplo do quadro(')); }\end{array}$} \\
\hline \multicolumn{5}{|l|}{ 12. Tem letra difícil de ler e entender; } \\
\hline \multicolumn{5}{|l|}{ 13. Evita situações que envolvem leitura e escrita; } \\
\hline \multicolumn{5}{|l|}{ 14. Tem dificuldade de identificar e/ou produzir rimas; } \\
\hline \multicolumn{5}{|l|}{$\begin{array}{l}\text { 15. Durante uma conversa, comumente, demora para se } \\
\text { lembrar o nome das pessoas, objetos, sentimentos ou } \\
\text { conteúdos escolares que já conhece (como se a palavra } \\
\text { estivesse na "ponta da língua"); }\end{array}$} \\
\hline $\begin{array}{l}\text { 16. Tem dificuldade para memorizar listas ou sequências de } \\
\text { informações (p,ex,, tabuada, meses do ano, dias da semana). }\end{array}$ & & & & \\
\hline
\end{tabular}

descreve a aprendizagem do(a) aluno(a) nos últimos 6 meses. É importante que você realize uma leitura completa do material, para depois responder a cada item.

1 Também conhecido por lousa em outras regiões do pais.

Por favor, responda também às perguntas abaixo em relação aos últimos 6 meses:

1. Em comparação aos colegas de turma, o desempenho desta(e) criança/adolescente em português está muito abaixo do esperado?

$\square \operatorname{Sim} \square$ Não

2. O aluno vem recebendo algum tipo de reforço/apoio ou adaptação pedagógica na escola?

$\square$ Sim $\square$ Não

3. O aluno vem evoluindo consideravelmente (exemplos: atualmente já consegue ler textos mais extensos e compreender; e/ou diminuiu, significativamente, o número de erros ortográficos; entre outros) nas suas habilidades de leitura e escrita?

$\square$ Sim $\square$ Não

4. Espaço para realizar observações que considerar pertinentes: 


\section{MATERIAL SUPLEMENTAR}

Este artigo acompanha material suplementar.

Tabela Suplementar 01. Cargas fatoriais do ELE

Tabela Suplementar 02, Consistência Interna valores do Coeficiente Alpha

Figura 1- material suplementar: Distribuição dos escores do ELE na escala de habilidade calculado pela TRI

Este material está disponível como parte da versão online do artigo na página: http://www.scielo.br/codas 